\title{
A Business Processes Model for the Integration of Over-The-Top Platforms in Communications Service Providers Operations
}

\author{
Carolina Fernandes ${ }^{1 *}$, Rui Cruz ${ }^{2}$
}

\author{
${ }^{1}$ Instituto Superior Técnico, Universidade de Lisboa, Lisboa, PORTUGAL \\ *Corresponding Author: carolina.c.pereira@outlook.pt
}

Citation: Fernandes, C. and Cruz, R. (2021). A Business Processes Model for the Integration of Over-The-Top Platforms in Communications Service Providers Operations. Journal of Information Systems Engineering and Management, 6(1), em0131. https://doi.org/10.29333/jisem/9570

ARTICLE INFO

Published: 16 Jan. 2021

\begin{abstract}
The problem between Over-the-Top (OTT) and Communications Service Provider (CSP), namely for multimedia streaming services, is not new and has been a topic much discussed in the TM Forum. At time of writing, a technical solution that would benefit both parties has not yet been presented. With the existing model (no operational integration), both OTTs and CSPs are dissatisfied. This paper proposes a technical solution that can be beneficial to both parties and can potentially solve the existing conflicts. The solution presents the high-level design of Business Processes to integrate OTT Service Platforms in CSP Operations. Some hypothetical, but close to reality scenarios were defined in order to show how the model could be applied. The design of the model incorporates information and suggestions collected from Portuguese CSPs. To evaluate the accuracy of the design methodology, a set of interviews were conducted with industry experts in the sector, who evaluated each process model of the solution. A set of surveys from end users of those types of services was also conducted, in order to understand their opinions about the ideas used for the construction of the model.
\end{abstract}

Keywords: Over-the-Top (OTT), Communication Services Provider (CSP), TM Forum, Design Science Research Methodology (DSRM), business processes

\section{INTRODUCTION}

In the past, Telephony and Connectivity operators (Telcos) were in full control of the few services they provided, technically managing their fixed-line and mobile radio communications infrastructures, basing their business models on long-term infrastructure investments that were financed through usage-based services, recurring subscriptions and connection commissions (e.g., leased lines). The changes that this industry has undergone after deregulation in all countries was due to both direct competitors and from services provided over the Internet by new "unsuspected" players, the so-called Over-the-Top (OTT) providers.

The proliferation of IP-based networks made it easier to decouple the Application and Network layers, making life easier for those OTT providers to deliver their digital services, contents and applications directly to end consumers, competing against similar services provided by the Telcos and jeopardizing their traditional business models, therefore bringing huge challenges and even threats to the survival of most Communications Service Providers (CSPs) (Czarnecki and Dietze, 2017; Portal et al., 2015). In truth, most of those CSPs, are evolved Telco companies that typically invest enormously, not just in their communications and Information Technology (IT) infrastructures but also in a solid Support Services (SS) architecture, i.e., Business Support Systems (BSS) and Operations Support Systems (OSS), able to cope with the evolving changes in digital operations, as they try to handle more "intelligent" automation, orchestration, redundant operations, flexibility, and autonomic principles, namely for Fulfillment and Assurance areas of those services, so that they can thrive in this new Digital Services future (Orange and Huawei, 2017). Since OTTs deliver their services over the Internet, they make use of the CSPs infrastructures up to their end-users, without incurring in any type of costs or be subject to regulations associated with them. CSPs are therefore seen as mere "bitpipe providers" because they do not "know" or control the type of services, contents and applications arriving from the Internet that their networks carry to their own customers. From the OTTs side, offering services over the Internet (best-effort networking) usually provides just enough quality, and so, even in a "Net Neutrality" ecosystem, they still cannot guarantee adequate quality for professional communications such as those in E-health, or ultra-high-definition multimedia streaming. From the CSPs side, the increasing amount of data that passes through their infrastructures, ultimately tends to create congestion, compelling them to build, upgrade and expand their networks at a faster pace, which in turn bring even more OTT traffic. The CSPs, although trying to compete with OTTs by widening their Service Portfolio, are still deeply worried because these OTT companies offer services that 
are extremely similar to theirs, and pose a credible and quantifiable threat to their revenues. To be able to turn the tables, CSPS have begun to realize that the time has come to invest strongly and increasingly in new applications, process automation, network infrastructures and customer care in order to differentiate them from competition (other CSPs, as well as OTTs).

In this basis, a structured transformation of organization, processes, applications, data and network technologies is an essential factor for success.

In our study the focus is on multimedia streaming services, that are strong favourites for the "on demand" experience and the main challenge to CSPs. This research work, takes as foundation the developments being made by the TeleManagement Forum (TM Forum, www.tmforum.org), that specifies a set of best practices and standards and provides a Framework Model that is acknowledged, well known, as well as contributed by the entire telecommunications industry, and can be seen as the de facto standard (Czarnecki and Dietze, 2017; ITU-T, 2007) and blueprint for the business operations, IT systems and ecosystems of CSPs. Currently, the TM Forum is deliberating on how to give an answer and find a balanced and well-adjusted solution to these setbacks that are directly affecting both CSPs and OTTs.

The journey has just begun for CSPs and OTTs: they have to work together and reach an agreement that will be beneficial for both parties, to meet the challenges that technology development brings. In essence, the problem is that current BSS/OSS of CSPS do not yet include a joint model with external OTT providers and their corresponding Service Platforms. To find a solution, it is necessary a digital operations transformation capable to provide orchestration and automation principles for the Fulfilment and Assurance areas related with services offered by the OTTs, but in partnership with the CSP.

The purpose of this work is to present the design of a future SS technical integration model between CSP and OTT providers, allowing to combine those partners and their products into the BSS/OSS processes. To achieve that goal, a Design Science Research Methodology (DSRM) was followed in this work (Peffers et al., 2008).

This work was presented on the 15th Iberian Conference on Information Systems and Technologies (CISTI) (Fernandes and Cruz, 2020). This work was supported by national funds through FCT, Fundação para a Ciência e a Tecnologia, under project UIDB/50021/2020.

The remaining of this document is structured as follows: Section Research Methology describes the methodology that was followed, Section Fundamental Concepts and State of the Art provides a theoretical background; Section Related Work describes some related works; Section Proposed Model describes the artefact and the elements that support it; Section Demonstration Scenarios describes the demonstration scenarios; Section Evaluation assesses the solution, and Section Conclusions draws conclusions on the research work.

\section{RESEARCH METHODOLOGY}

As a problem-solving model, DSRM recapitulates the creation and assessment of a ground-breaking IT "design artefact", in order to attend to an important organizational problem. Constructs, models, methods and instantiations are considered design artefacts. As a result of the feedback offered by assessment phases, this iterative approach aims to enhance both the quality and process of the design artefact, looking for its veracity (justified theory) and utility (effective artefacts) and evaluated when it comes to its practical implications (Hevner et al., 2004). This methodology (Peffers et al., 2008) for the production and presentation of Design Science Research (DSR) in Information System (IS), is made up of six phases: (1) Problem identification and motivation, describing and identify the research issue, justifying the solution's importance; (2) Defining the solution's goals, deriving the goals from the problem definition, taking into consideration what is possible and feasible; (3) Design and development of the research artefact, once determined its desired functionality and architecture; (4) Demonstration, to show how to use the artefact, which can be accomplished through experimentation, simulation, case study, proof or other applicable activity; (5) Evaluation, by observing and assessing how well the artefact supports a solution to the identified problem; and, (6) Communication of the problem and its value, the artefact, its functionality and originality, the thoroughness of its design and its effectiveness to relevant audiences.

\section{FUNDAMENTAL CONCEPTS AND STATE OF THE ART}

This section, presents and discusses the main concepts and technologies, related and addressed in this work.

\section{OTTs and CSPs Strategies}

With the emergence of OTT, there was a turn from traditional telephony to OTT Communications and a replacement of Short Message Service (SMS) by OTT applications such as WhatsApp (www.whatsapp.com). Traditional Broadcast services (radio, television, news, audio and video transmission) are also being "replaced" by multimedia streaming "apps" such as those offered by Netflix (www.netflix.com/pt/), HBO (hboportugal.com), Spotify (www.spotify.com/pt/), FOX Play (www.foxplay.pt), Mubi (mubi.com), Tidal (tidal.com) and Qobuz (www.qobuz.com), as young adults watch less linear TV, preferring Internet-based streaming services (Richter, 2017). It is therefore obvious that CSPs are facing a massive challenge in their businesses, having to react quickly in order to keep up with today's technological advances and this competition to their traditional core business.

The business models of OTT Services rely deeply on competition, the market, and customer segmentation, and are based upon the following typical revenue sources (Hessler, Baldry, and Steingröver, 2014; Steingröver, Cardozo Larrea, and Zhelev, 2019): (1) Subscription: Customers pay a periodical fee to benefit from the service; (2) Usage (or Transaction): by metering the usage or each 
transaction; (3) Advertisement: where Suppliers sell different advertisements based on costumers preferences or habits; (4) Donation: in platforms (such as Wikipedia) funded by donations (Crowd Funding); (5) Freemium: where customers may benefit freely from the basic features, but have to pay to access premium or convenience features; and, (6) Monetization: where Suppliers pay users for disclosing private information.

As described by Steingröver, Cardozo Larrea and Zhelev (2019), it is possible to identify eight strategic options for a CSP when dealing with OTT players:

The Zero Option: No action is taken against OTTs. OTT services are seen as a disadvantage to flat-rate contracts, but as beneficial to the "pay as you use" contract models;

The Defence Strategy: This strategy has two approaches "Data Capping" and "Network Slicing";

The Attack Strategy: This strategy normally leads to blocking or throttling traffic or to emulate OTT Services;

The Cooperative Strategy: Three approaches can be considered: "Product Partnership", "Sponsored Data” approach, acquisition of OTTs.

A "do nothing" strategy will typically not work and would result in CSPs definitively becoming just bit-pipe providers. Many of those attack strategies are blocked by current regulations in most countries. Simple cooperation strategies are good short term strategies, but may not differentiate much in competition if many CSPs simply follow that approach. Therefore, defence strategies with data capping in the short term, and network slicing in the medium term, might be the recommendable way for most.

For CSPs preferring partnership models, the cooperation can enable and simplify the creation of new revenues if they sell premium OTT services and agree on some form of revenue sharing (Limbach, 2014). Contributing to that interest is the growing demand for high service quality, namely in multimedia streaming services. This is an important topic in our analysis related with the principle of "Net Neutrality", which is that any Internet Service Provider (ISP)/CSP must treat all traffic in their networks likewise (i.e., not discriminating or charging differently) regardless of its origin, destination or content.

Nevertheless, though rarely reported, it is common practice for CSPs to "regulate" the bandwidth in their networks in the case of extreme volumes of data transfer from OTTs, e.g., streaming of Ultra High Definition (UHD) multimedia contents, by prioritizing their own traffic or limiting (throttling) interconnect Internet traffic, reducing therefore the quality of OTT services.

A strategy based in collaboration and mutual cooperation is therefore the approach considered for the solution in this work, in order to benefit both parties.

\section{Support Services: OSS/BSS}

OSS and BSS are used to support all the telecommunication services of a CSP (Mallu and Ezhilarasie, 2015). BSS corresponds to customer-oriented business operation processes, such as Order Management or Customer Management. OSS correspond to infrastructure-oriented operations (Networks, Services Platforms and support functions) from service Fulfilment processes for provisioning and activation (including network configuration), and service Assurance processes (including monitoring and problem management). OSS are designed to help reduce the overall operating costs and improve efficiency, through automation of the key workflows of the operations.

\section{TM Forum's Frameworx and the Enhanced Telecom Operations Map (еTOM) Standard}

The TM Forum is a neutral, non-profit member organization that promotes collaboration and collective problem-solving in order to maximize the success of (tele)communications and digital services providers and their supplier ecosystem (The TM Forum Website, 2020). The TM Forum community was responsible for creating the Frameworx (TM Forum, 2019c), a suite of best practices and standards that enable a service-oriented, highly automated and efficient approach to business operations.

The Frameworx is composed by a suite of components, such as the Business Process Framework (eTOM) (ITU-T, 2007), the Application Framework (TAM) (TM Forum, 2019a) and Information Framework (SID) (TM Forum, 2019d), together with the Integration Framework, Business Metrics (TM Forum, 2019b) and Best Practices. The Frameworx delivers standardized Business Metrics that the industry has embraced and allows for benchmarking, as well as an assembly of interfaces and Open Application Program Interfaces (APIs) that allow integration across systems and platforms. Companies also benefit from Frameworx since it also helps implement and use top standard and management processes to guarantee ongoing conformance.

еTOM designates the interaction of the processes between different stakeholders in the telecommunications value chain and also contains cross-functional views that show how process components span horizontally across a company, allowing for superior detail in decoding the interaction process throughout the organization. According to the eTOM model, all company business processes are divided into three vertical functional groups in its first level of specification: Strategy, Infrastructure \& Product; Operational Management and Enterprise Management (Korzachenko and Getman, 2010): The Shared Information/Data Model (SID) delivers a reference model for all the information that is needed to implement the Business Process Framework processes, reducing system and services integration complexity, development and design by offering an off-the-shelf information model that can be quickly adopted by all parties (TM Forum, 2019d). The Telecom Application Map (TAM) offers a common-sense grouping of applications, defining what functionality is offered by each application. It also allows for a mutual language between communities who specify, acquire, design, and sell OSS and OSS solutions, making it possible to understand each other's viewpoints (TM Forum, 2019c). The Integration Framework is a set of criteria that supports the interoperability in-between applications outlined in TAM via TM Forum interfaces. The interfaces are defined in terms of the SID's entities/attributes, and the necessities for the interfaces from a business process perspective, which comes from eTOM. Best Practices provide useful and practical tools that balance Frameworx and help to improve end-to-end services management across complex, multi-partner environments. 
For this study the focus was on the eTOM component of Frameworx, which is used to describe and position OSS and BSS systems and processes in relation to one another.

\section{Modeling Languages and Tools}

The modeling languages and tools that were used for the design of the proposed solution, were Business Process Model and Notation (BPMN), Unified Modelling Language (UML) and Archimate. BPMN (Fettke, 2011; OMG, 2014) provides a graphical notation for business process specification, based on a flowcharting technique very similar to the activity diagrams of the UML. Its main objective is to support business process management by providing a notation understandable by all business stakeholders while also being able to represent complex process semantics. UML is a modeling language for software engineering that provides a standard way to visualize the design of a system. Archimate is an enterprise architecture modeling language and a standard (OMG, n. d.). ArchiMate distinguishes itself from other modeling languages such as UML and BPMN by its enterprise modeling scope. Thus, Archimate not only supports the description of business processes but also organizational structures, information flows and IT systems and infrastructures.

\section{RELATED WORK}

In this section, the works "Next Generation Telco Architecture", by Deloitte and the "Future Operation Support Services" by Huawei and Orange are presented and discussed.

\section{"Next Generation Telco Architecture"}

This theoretical Telco architecture is founded on an Application Program Interface (API) Fabric model that depends on microservices, "softwarized" infrastructures and big data abilities that determine the features of the current IT architectures of the Telco industry (Cerulli and Green, 2017), justified by the fact that the continuing digital transformations are not capable of supporting today's IT architectures because they are "intricate, inflexible and retain reduced amounts of automation, since their procedures result from a large number of merges and acquisitions". The author also defends that operators should embrace the IT reference model developed by OTTs in order to solve current IT architecture errors, therefore achieving an architecture founded on the use of microservices, APIs, Big Data and cloud platforms, i.e., the BSS/OSS API Fabric Architecture.

OSS/BSS are distributed into smaller components within this architecture, according to the microservices paradigm or substituted by Software as a Service (SaaS) solutions. Each component has its functionality which is stable and reusable. Breaking down systems into smaller services allows the same technical features to be reused in diverse business processes. Microservices and the SaaS components interact through APIs. Business processes are achieved through a choreography model, where interactions are directly managed by microservices. Accordingly, the communication layer is implemented using a simple "message bus".

The author points out that Data will be increasingly consolidated into a centrally managed Information Architecture (IA) in the Data Tier Layer, by means of a mutual and combined information model. The IA is responsible for offering access to information through different functions. Through standard APIs, access to information (offered as a service) will be implemented, which avoids the need to understand low-level data constructions. When it comes to the Infrastructure Layer, as so to satisfy business requirements that need rapid and agile deployment, the IT infrastructure must grow in order to adopt cloud technologies, such as Infrastructure as a Service (laaS).

Big Data and analytics skills are consolidated into centralized systems when it comes to the Big Data Layer. Analytics will interact with the API Fabric environments, by means of real-time analysis and pattern recognition, therefore prompting the start of optimization workflows. Using Big Data analysis, Analytics will support developments of the customer experience, enabling pattern recognition and reacting in almost real-time, thus starting the implementation of distinct business processes.

The author concludes that new applications can be developed using typical building blocks thanks to Platform as a Service (PaaS) solutions, with building blocks libraries enriched with reusable components.

\section{"Future Operation Support Services"}

The strategic plan described by Huawei and Orange addresses digital transformation and presents a common architectural vision that points out how to design agile Future OSSs. This OSS defines an architecture framework that provides a common digital platform for network and service administration and is made up of several domains (Orange and Huawei, 2017), namely: (1) Cloud/PaaS: The Future OSS cloud-based platform; (2) Design Systems: enabling DevOps and model-driven design; (3) Support Systems: linking the Future OSS Design-Time and Run-Time via Dynamic Inventory, Catalogue, Policy Management, and API Management; (4) Orchestration: automating and accelerating all actions necessary to provide end-to-end service management for cost effective hybrid Fulfilment and Assurance. Data Collection and Analytics modules within Orchestration extend this support to allow intelligent closed loop operation; (5) Fulfilment: providing the link with existing OSS/BSS implementations, turning business requests into solutions; and, (6) Assurance: guaranteeing quality of service through dynamic operations and automation of manual, time consuming tasks.

\section{Discussion}

Conceptually similar to the architectures presented in Cerulli and Green (2017) and Orange and Huawei (2017), our model also aims to answer to the future needs of telecommunications businesses and proposes to function as a platform component that 
integrates another platform/service, that is, the OTT would function as a Service Platform, being integrated in the CSP as any other Service Platform. Cerulli and Green (2017) claims that operators must adopt an architecture based on the use of APIs, microservices and Big Data. The model we propose advocates the same principles, considering that the operator's platform interacts with the external Partner platform through APIs. As each component of the API Fabric model is stable and easy to reuse, our model also aims to include this feature.

The model we propose in this work also aims to demonstrate how to design a swift future OSS based on the eTOM framework, focused on the processes of Fulfilment, Assurance and Billing.

\section{PROPOSED MODEL}

Based on the analysis of the related work and on the identified research problem, the following objective was established for the proposed model:

To develop a model that allows CSPs to work together, i.e., a model that allows the integration of OTTs Service Platforms on CSPs platforms.

In order to obtain a model as accurate as possible, several iterations were performed.

In an initial phase, the theme was validated by the two largest national operators, Vodafone Portugal and NOS Comunicações, and by an OTT provider, Netflix, in order to establish the potential of the subject and to identify their interest in this great challenge. The opinions were favourable regarding the concept and both operators showed interest in validating the proposed model.

The following sections briefly present the AS-IS and TO-BE for the service delivery model of the operators, as well as the design (in high-level detail) of some of the key processes.

\section{AS-IS Operators' Service Distribution Model}

CSPs provide three types of basic services to their customers: telephone (voice communications), Internet (access) and TV (linear broadcast-type and on-demand). These services are commonly sold together in Product bundles. Television and telephone services over fixed access network, have signal priority over Internet services and therefore service quality is only "guaranteed" for telephone and TV.

As mentioned previously, it is through the Internet that OTT providers reach the final customer and, as such, they cannot guarantee service with quality for their products (unless a business agreement is established with the CSPs).

In an ideal world, the service quality problem would be solved if the Internet service had an end-to-end priority, at least equal to the other services. However, this is not what happens and, as such, it was necessary to review the current model of CSPs in order to be able to understand which processes could be improved.

\section{TO-BE Operators' Service Distribution Model}

To allow OTTs to distribute their services with guaranteed quality, and at the same time enabling the CSPs to invest further in their infrastructures assured that they will be compensated by ideally "shared" revenues from OTT services, an alternative model for services distribution was designed. Considering, for simplification, the case of multimedia content distribution, if the OTT services would be treated at the same priority level as the TV service, the service quality problem would be eliminated as the CSP has absolute control over the quality parameters in their access networks. In addition, Internet service overload due to video and music streaming from OTTs would eventually not occur, since these services would be distributed through a resource controlled channel.

\section{New Models and Processes}

In this section we will present the various processes and models developed in order to support the alternative model of a CSP service delivery.

\section{Product definition and provisioning diagram}

The development of a flexible data structure for the definition and provisioning of telecommunication products is based on common approaches of the manufacturing industry (Czarnecki and Dietze, 2017). The solution (Figure 1) consists of product (service bundles with both technical components and commercial specifications), service (OTT services providing features, which are set independently of their technical achievements) and resource (the technical achievements of features) corresponds to the Service Type. The customer buys a product with certain defined functional parameters. The purchase of a product is processed into a Customer Work Order, which is then unbundled into one or more Service Work Orders. These Work Orders have an associated "Customer_id" that identifies the consumer who triggered the Work Order. 


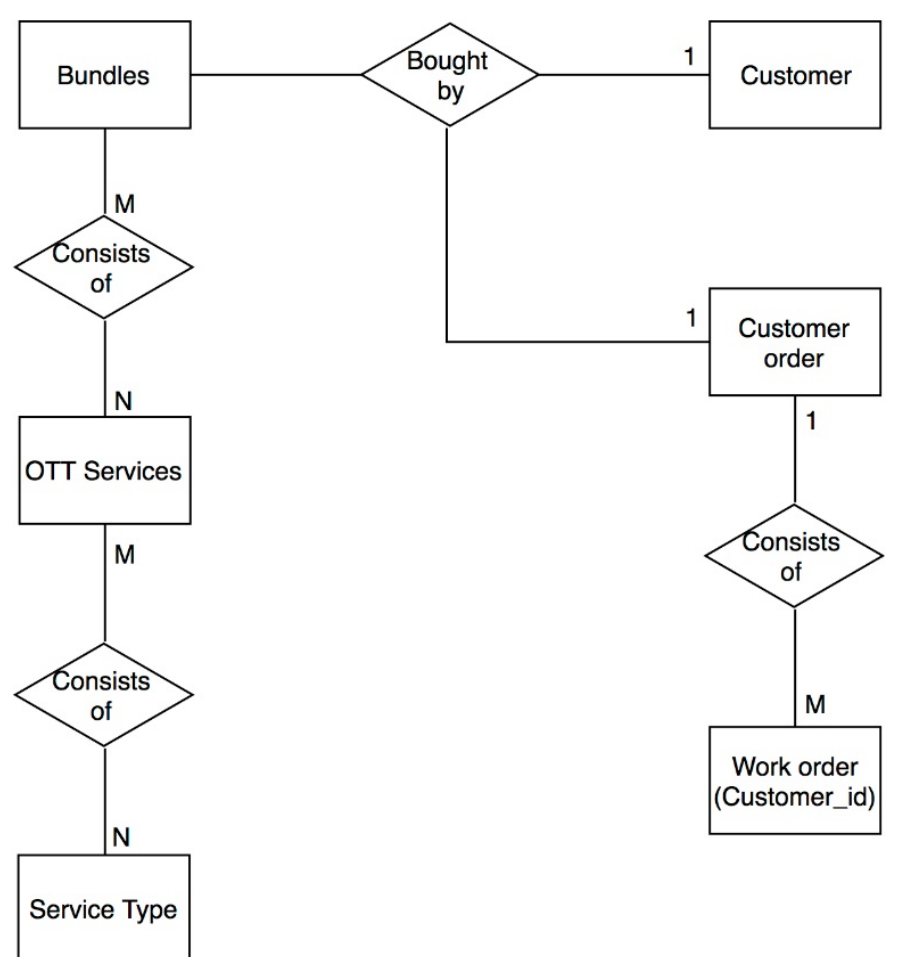

Figure 1. Flexible data structure for product definition and provisioning. Adapted from (Czarnecki and Dietze, 2017)

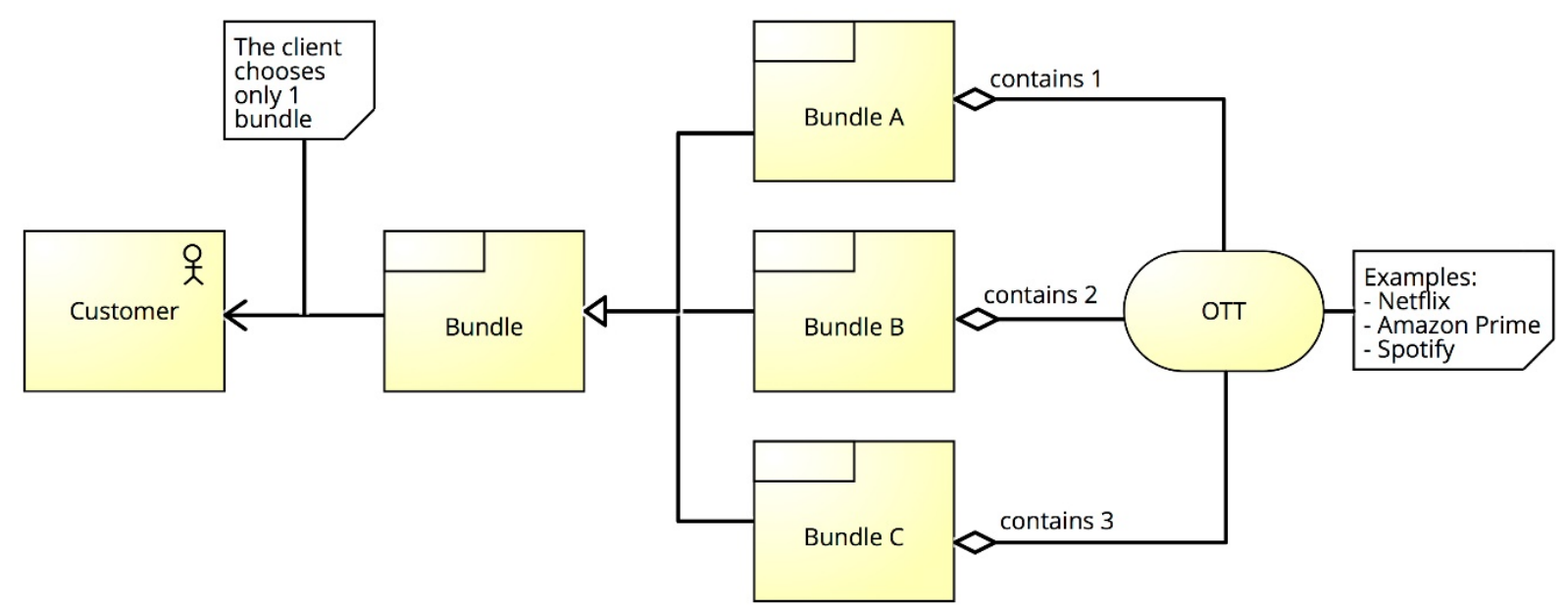

Figure 2. Product Viewpoint

\section{Product viewpoint - Bundles diagram}

The product viewpoint presented in Figure 2 aims to represent the options that the customer has when joining OTT services offered by a CSP.

By way of example we define three bundles with different content:

- Bundle A, with this bundle the customer has the right to choose one OTT service;

- Bundle B, the customer can choose two OTT services (e.g., a Video service and a Music service);

- Bundle C, allows the customer to choose three OTT services.

For all the bundles there is a list of available OTTs, from which customers can choose the ones they want. This list is defined by the CSP, according to the partnership agreements between the CSP and the OTTs.

\section{Membership}

The process begins when a Party (Individual or Organization) wants to subscribe to a bundle that includes OTTs. The Party is checked in order to determine if it is already a Customer. In the case of being a new Customer of the CSP, a customer file and a 


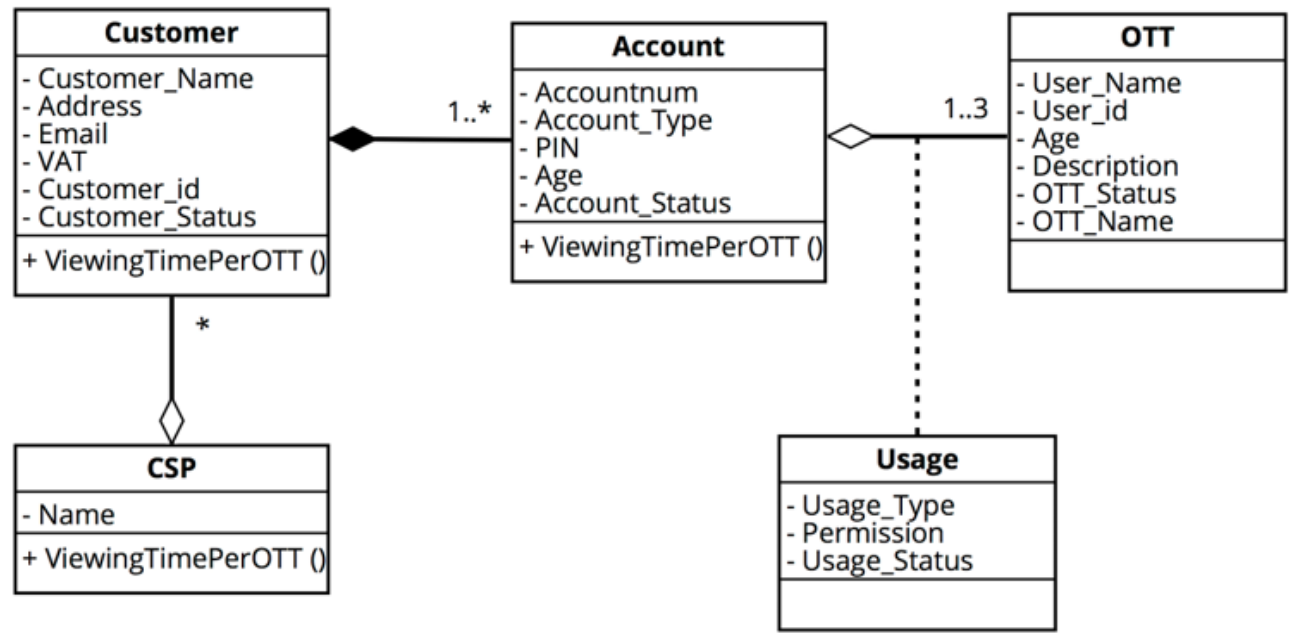

Figure 3. Class Diagram

"Customer_id" are created. Once the account settings are finalized, it is possible to subscribe to services to be provisioned for each account of the Customer.

\section{Class diagram}

The Unified Modelling Language (UML) diagram of classes represented in Figure 3, aims to outline the associations between the several classes, as well as their methods and attributes.

In Portugal there are four main service providers (CSPS): NOS, Vodafone, Altice/MEO and NOWO. These CSPs are represented by the class "CSP". A CSP aggregates multiple customers ("Customer" class) which, in turn, have one or more accounts associated to their customer file.

Each account can be associated to 1, 2 or 3 OTTs. This class has the following attributes:

- An account number ("Accountnum") that allows to uniquely identify the User of each account.

- A type ("Account_Type") that distinguishes the type of account: child or adult.

- A PIN code that allows the customer to block undue access to created account parameters;

- An age ("Age") that enables the service to recommend content that is appropriate for each age group.

- A status ("Account_Status") that allows to know the status of each account, i.e., whether the account is active, blocked due to non-payment or disabled.

The "Usage" class represents the relationship between the "OTT" and "Account" classes, allowing to check the permission that a certain account has for accessing a certain OTT. The OTT class allows to determine the status of OTT account, i.e., whether the account is active, blocked for non-payment or disabled. The "ViewingTimePerOTT" method allows to compute the viewing time per OTT for each account, client and CSP, respectively. The "Customer" class has attributes that belong to the customer files created when joining one of the bundles.

\section{Reference process flow "Order-to-Payment"}

The "Order-to-Payment" process (Figure 4), situated at Level 3 of the model, is responsible for the commercial processing of a Customer Order, the Provisioning and Activation of the Services and the subsequent Billing of consumed services. 


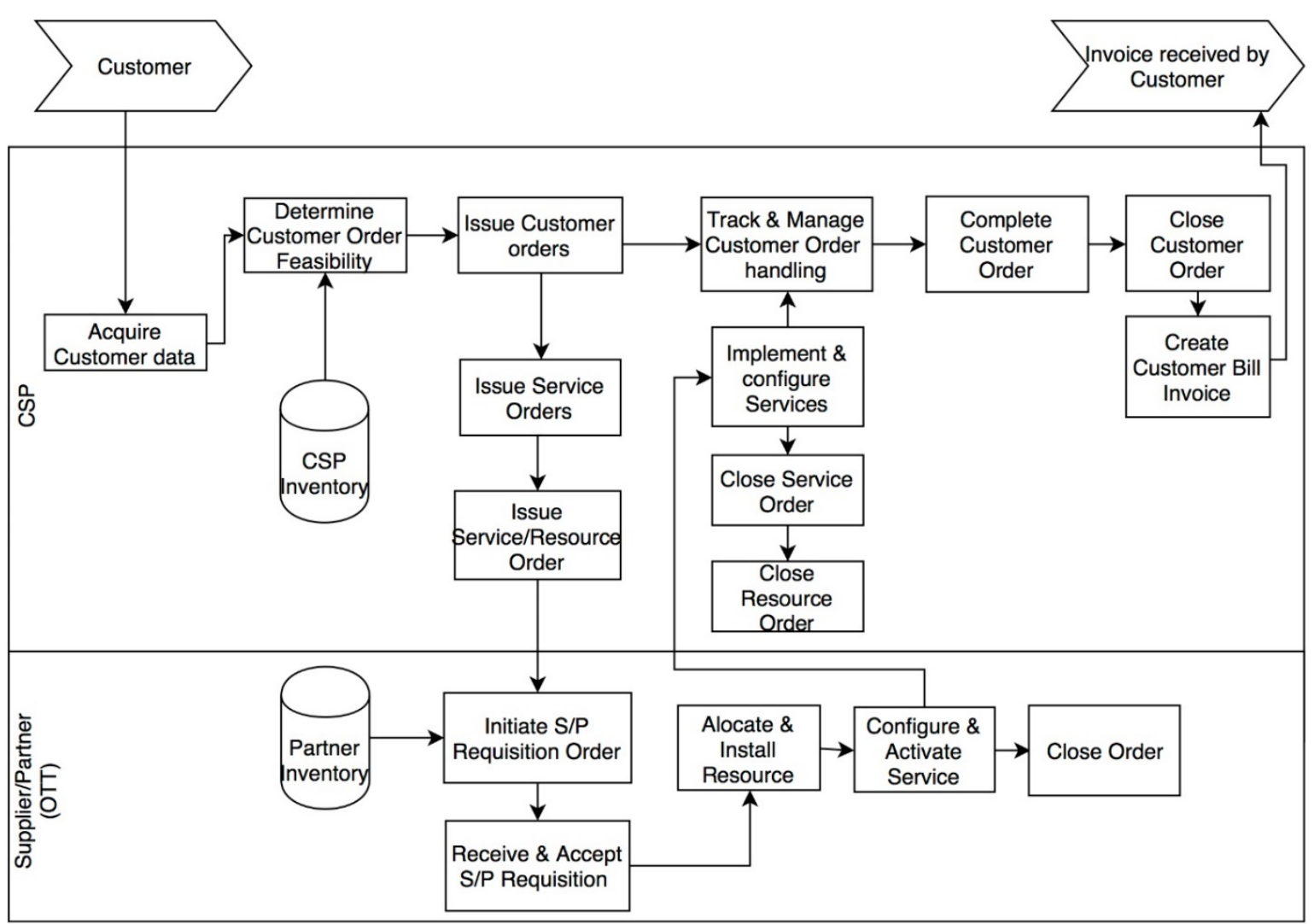

Figure 4. Level 3 - Process elements in Order-to-payment. Adapted from: (TM Forum, 2012)

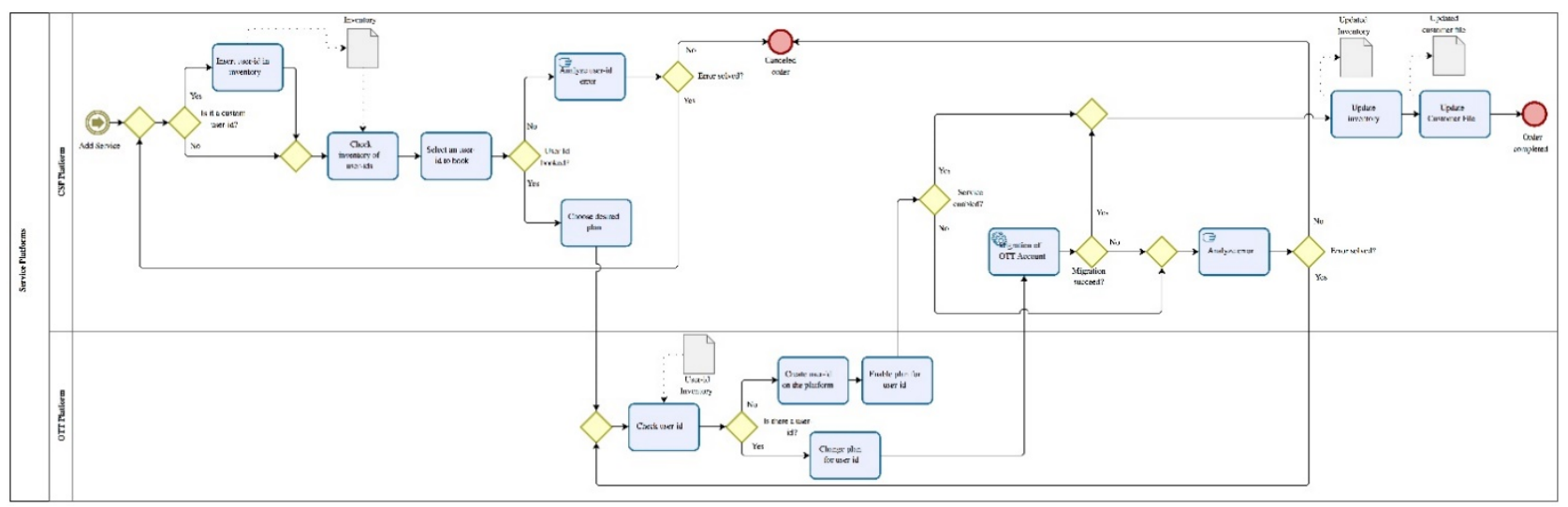

(a)

Figure 5. Level 4 - "Order-to-Payment” Service Processes (Level 4): (a) Add Service; (b) Change Service; (c) Remove Service.

The various types of activities that a Customer can request from the CSP are typically streamlined as Adding, Changing or Terminating services. These "Order Processing" activities, detailed at Level 4 of the model, are therefore implemented as Work Orders of type "Add Service", "Change Service" and "Remove Service", as illustrated in Figure 5.

As for the processes design, it is assumed that a "Customer" already exists (was created) on the CSP platform, that is, the "Customer_id" is already known and was validated for the Work Order. It is important to take into consideration that the data object represented in these processes as "inventory" corresponds to the CSP Service/Resource Inventory subsystem, which contains all the "User_id"s available for the CSP to assign to their Customers. These "User_id"s are defined and distributed by OTTs to CSPs. The data object represented as "User_id inventory" contains all the OTT "User_id"s, that is, the "User_id"s distributed by the CSPs, as well as the "User_id"s created directly on the OTT platform.

The Customer Work Order process starts when a Customer requests one of the following situations:

- Add Service: When a Customer wants to add a service, an order of type Add Service is created, as illustrated in the process flow of Figure 5a:

- The CSP platform checks whether the "User_id" is custom or "stock". This situation occurs, for example, when a customer had previously subscribed services directly of an OTT and wants to migrate the account to the CSP, in order to preserve her/his preferences and usage history. 


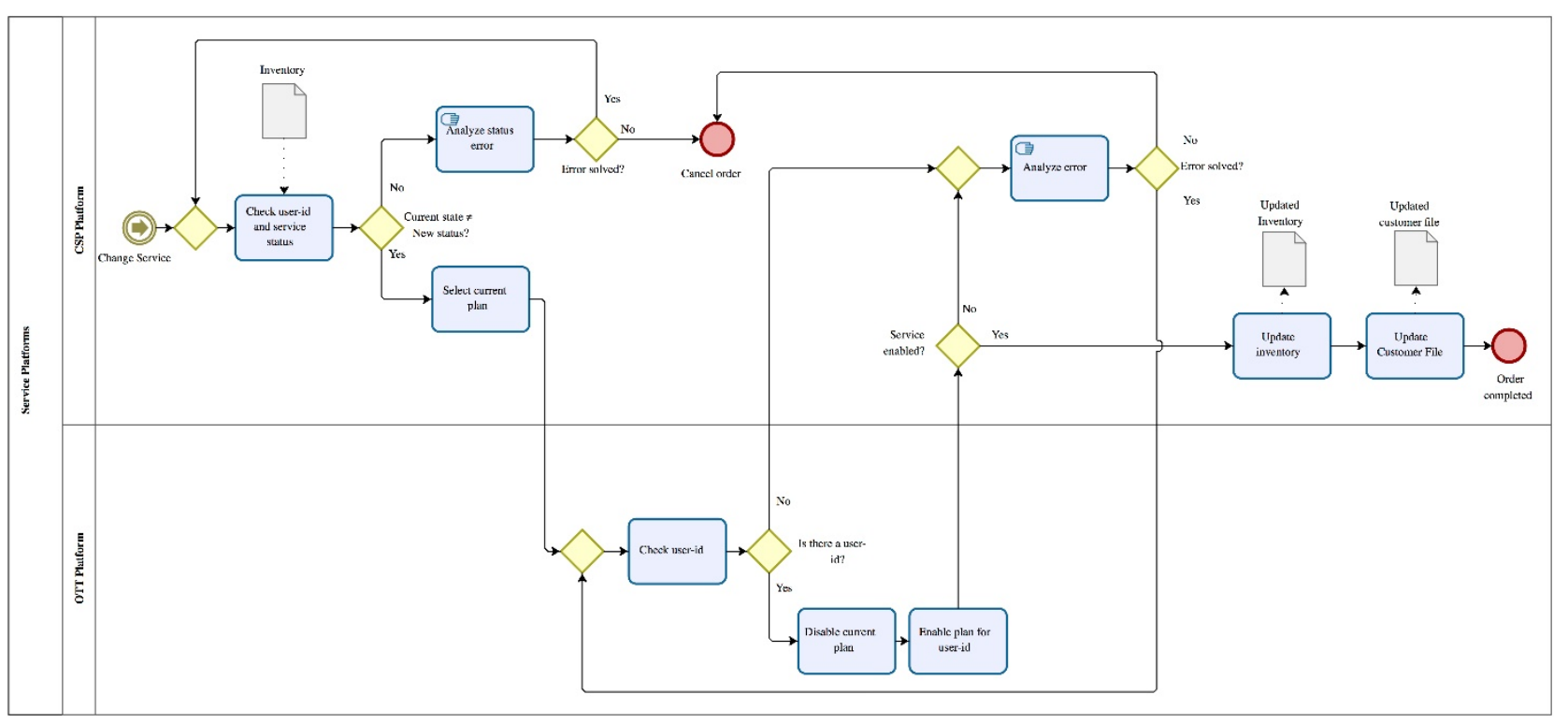

(b)

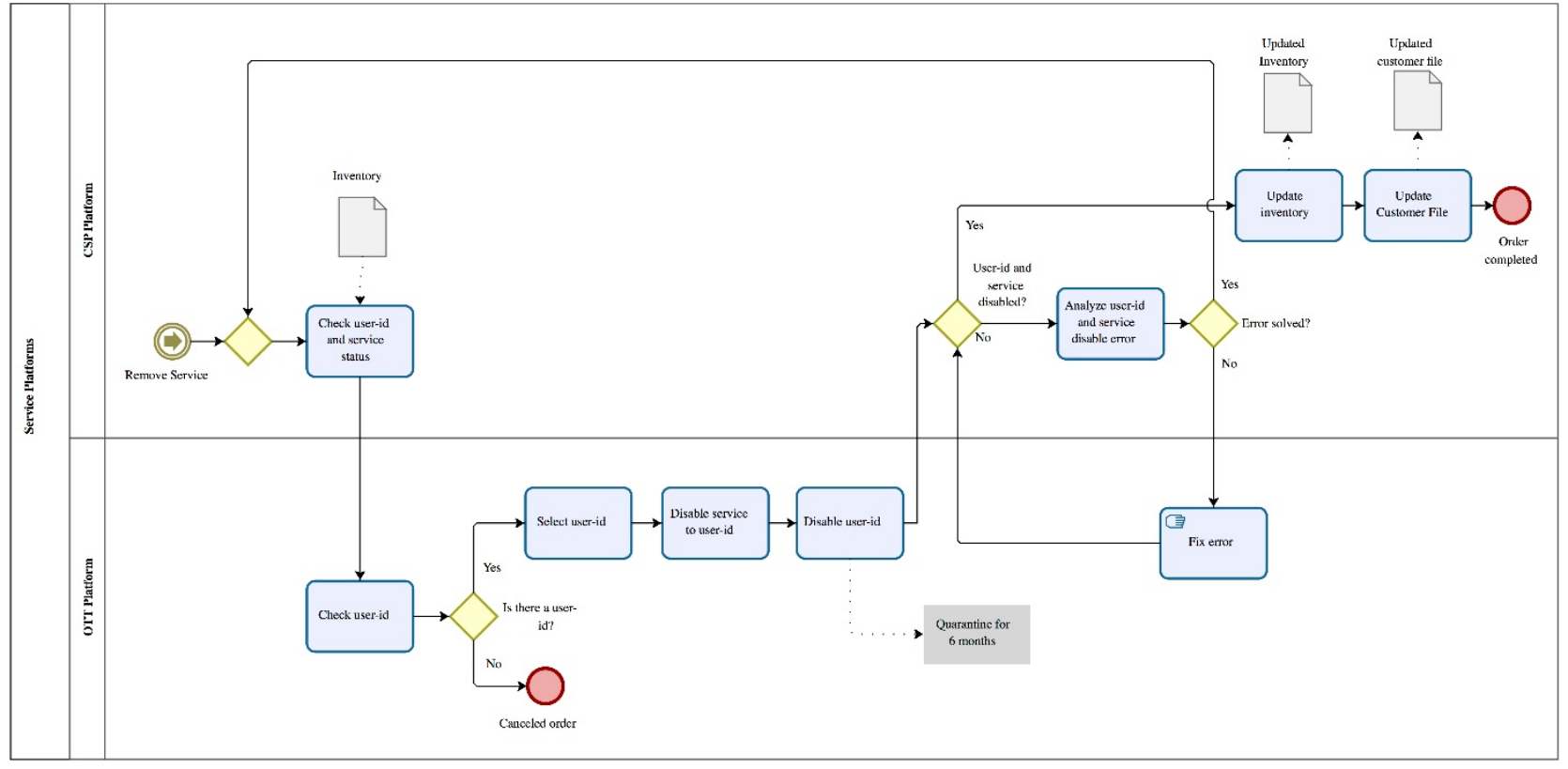

(c)

Figure 5. Level 4 - “Order-to-Payment” Service Processes (Level 4): (a) Add Service; (b) Change Service; (c) Remove Service.

- The "User_id" status is then verified in the Resources Inventory, which, when presented as "reserved", allows to associate it with the desired product/service plan.

- After selecting the desired plan for the OTT platform, an external order request is triggered to the Partner OTT platform.

- If the "User_id" exists on the OTT platform, the plan for the service subscribed with the CSP updates the OTT Service Plan and triggers an account migration process to the CSP platform. If the migration succeeds, the Resources Inventory and the customer file are updated and the order is complete.

- Change Service: When a Customer wants to change a service, an order of type Change Service is created as illustrated in the process flow of Figure $\mathbf{5 b}$ :

- In the CSP platform, the "User_id" and the service state in the Resources Inventory are verified, and later confirmed whether the current state is different or not from the new state, in order to activate the desired service state.

- If the service is activated, the Resources Inventory and the customer file are updated and the order is complete.

- Remove Service: When a customer wants to unsubscribe to a service, an order of type Remove Service is created, as illustrated in the process flow of Figure $5 c$ :

- In the CSP platform, the "User_id" and state of the service are verified in the Resources Inventory, and subsequently verified on the OTT platform. 
- If it exists on the OTT platform, then the service associated with it is disabled. As such, the "User_id" is deactivated since there is no service associated to it. This "User_id" is however quarantined for a period of 6 months, to prevent its usage for other customers, and to also allow for reactivation to the same customer account.

- After deactivating the "User_id"' in the CSP platform, it is verified that the service and "User_id" are disabled, after which the Resources Inventory is updated with the information that this "User_id" is quarantined for 6 months and the client file is also updated, thus completing the work order.

\section{Usage-to-Payment process}

The "Usage-to-payment" process, another Level 3 process, begins with the customer decision to subscribe to a product and ends with the payment for the used product.

Based on the contract, the use of the service requires a usage-based payment, which is related to the volume of data spent, the time of use of the service or number of accesses to the service, depending on what is agreed between the CSPS and the OTTs. The collection and rating of usage data is part of the technology domain. The diagram of Figure 3 represents the class "Usage" that intends to show how the accounting can be done through the defined attributes (simplified for this example).

\section{DEMONSTRATION SCENARIOS}

The Demonstration corresponds to phase 4 of the DSR methodology. Therefore, and in order to demonstrate the feasibility of this solution, the following hypothetical scenarios, but close to reality (as confirmed by the CSPs), were defined, in order to understand the real application of the designed model.

- S1: A user who was already an OTT customer and intends to migrate the OTT account to his CSP;

- S2: A new CSP customer who intends to contract a Product that includes OTT services;

- S3: A user that reduces the level of service, i.e., changes the OTT plan;

- S4: A customer that chooses to cancel the contract with a CSP and later decides to reactivate it.

For those scenarios, it is assumed that the OTTs and corresponding services are available in the Product Catalogue of the CSPs, and so, are the result of partnership contracts between both types of providers.

\section{EVALUATION}

The fifth phase of the DSR methodology corresponds to the Evaluation and aims to measure how well an artefact supports a solution to the problem by comparing the proposed objective with the observed results of the use of the artefacts in the demonstration. For this purpose, the artefact consistency was evaluated through case studies (the Demonstration). The accuracy of the methodology was evaluated through interviews with experts in the industry, and a set of surveys for users of these type of services, in order to understand their opinion about some ideas used in the construction of the model.

The survey obtained $\mathbf{3 0 4}$ responses with every region of Portugal represented, as well as various age groups. From the answers, $\mathbf{1 7 9}$ of the respondents already use at least one OTT service, of which $\mathbf{8 0 \%}$ use the service more than twice a week, and $\mathbf{8 5 \%}$ consider that the quality of transmission is one of the most important features. Regarding the integration of OTT services in the CSPs' platform, $\mathbf{8 2 . 6 \%}$ considered that it would be interesting to include OTT services in the CSPs offers, and $\mathbf{8 2 . 2} \%$ considered as advantageous to access OTT services from their TV. Finally, $\mathbf{7 5} \%$ agreed that a joint invoicing would be a good option.

The interviews were carried out with 5 specialists in the areas of Telecommunications, OSS / BSS, Service Platforms, Automation and Product Management. The interviewees belonged to national CSPs (NOS Comunicações and Vodafone Portugal), and the positions occupied were of Product Management Director, Director of Service Platforms, Operations Director and Automation Manager. For the interviews a script was used as, containing specific questions related to the processes (illustrated with the diagrams and process flows of the developed Model). This script consisted of 18 questions covering all the proposed processes. All the interviewees considered the research work as "Very Relevant" enumerating the reasons for that classification: The increase in bulk and presence of OTTs in network traffic can be a potential threat to the CSPs business. Since their growing presence, and the corresponding traffic, are not expected to decrease, it is a real and factual problem that has not yet a solution; OTTs services are dictating consumer habits and so, it is inevitable that they should/must be an integral part of CSPs' offerings;

Regarding difficulty in terms of resources and cost/benefit for the integration of an OTT service, the respondents answered that, technically, it would be equivalent to any other integration they already make and that the benefit would be the differentiation in the portfolio of services offered. Regarding an implementation of a distribution model together with OTTs that uses streaming mechanisms but ensures quality of service in the Access network, the respondents answered that it would be easier to prioritize the OTT's traffic with whom they had established an agreement and thus guarantee the desired OTT service quality.

\section{CONCLUSIONS}

The main objective of this work, was to develop a technical model to allow the integration of Over-the-Top (OTT) services on Communications Service Providers (CSPs) platforms. 
To conduct this research, a Design Science Research (DSR) methodology was followed, starting with the clear identification of the problem, i.e., nonexistence, yet, of a joint model with OTT providers as Partners of CSPs. In order to obtain a model as accurate as possible, several iterations in the design were performed. To demonstrate the feasibility of the developed model, some case studies were created considering hypothetical, but close to reality scenarios, in order to understand its real application. Finally, to evaluate the accuracy of the methodology, interviews with experts in the industry were conducted, together with a set of surveys with users of these types of services. From the results it was possible to conclude that the model meets the requirements and objectives formulated, and that it would bring advantages for both OTTs and CSPs, such as guaranteed support and maintenance of the OTT's proxied Service Platform nodes hosted at the CSP core network, and predictable infrastructure investments for the CSP, with the traffic under control and with assured service quality.

Research opportunities for future work include detailing the process models at level 3 and 4 of the eTOM framework, development of open APIs in partnership with OTTs and CSPs, and implementation of an experimental solution in partnership with a OSS/BSS solutions supplier.

\section{REFERENCES}

Cerulli, L. and Green, D. (2017) Next Generation Telco Architecture. Available at: https://www2.deloitte.com/content/dam/Deloitte/ it/Documents/technology-media-telecommunications/Next\%20Gen\%20Telco\%20Architecture_2017_final.pdf

Czarnecki, C. and Dietze, C. (2017) Reference Architecture for the Telecommunications Industry. 1st edn. Springer International Publishing. https://doi.org/10.1007/978-3-319-46757-3

Fernandes, C. and Cruz, R. (2020). A Process Model for the Integration of Over-The-Top and Communications Service Providers. In 2020 15th Iberian Conference on Information Systems and Technologies (CISTI), pp. 1-6. https://doi.org/10.23919/CISTI49556.2020.9140908

Fettke, P. (2011). Business Process Model and Notation. Business, 50(January), 170. https://doi.org/10.1007/s11576-008-0096-z

Hessler, M. A., Baldry, S. and Steingröver, M. (2014). The rise of OTT players: what is the appropriate regulatory response? 25th European Regional ITS Conference, Brussels 2014. https://doi.org/10.1007/978-3-319-77724-5_21

Hevner, A. R., et al. (2004). Design Science in Information Systems Research. MIS Q. Minneapolis, MN, USA: Society for Information Management and The Management Information Systems Research Center, 28(1), 75-105._https://doi.org/10.2307/25148625

ITU-T (2007) Enhanced Telecom Operations Map (eTOM). Geneva.

Korzachenko, O. and Getman, V. (2010). Improvement of Business-Activities in Telecommunication Enterprises by the eTOM Business-Process Structural Model Implementation. Scientific Journal of Riga Technical University. Computer Sciences, 42(1), 45-50. https://doi.org/10.2478/v10143-010-0040-8

Limbach, F. (2014). Cooperative service provisioning with OTT players: An explorative analysis of telecommunication business models. In. Brussels: International Telecommunications Society (ITS) (25th European Regional Conference of the International Telecommunications Society (ITS), Brussels, Belgium, 22-25 June 2014). Available at: http://hdl.handle.net/10419/101388

Mallu, L. and Ezhilarasie, R. (2015). Impact of Over the Top (OTT) Services on Telecom Services Providers. Indian Journal of Science and Technology, 8(February), 326-332. https://doi.org/10.17485/ijst/2015/v8i

OMG. (2014). Business Process Model and Notation (BPMN). Available at: https://www.omg.org/spec/BPMN/

OMG. (n. d.). Archimate. Object Management Group. Available at: https://www.opengroup.org/archimate-forum (Accessed: 9 June 2020).

Orange and Huawei. (2017). Future OSS - Providing the agility to support digital operations transformation of hybrid networks. Available at: http://carrier.huawei.com/ /media/CNBG/Downloads/Services/SolutionTopic/oss/OrangeHuaweiFutureOSS WhitePaperfinal.pdf

Peffers, K., et al. (2008). Design Science Research Methodology for Information Systems Research. Journal of Management Information Systems, 24(3), 45-78. https://doi.org/10.2753/MIS0742-1222240302

Portal, J., et al. (2015) Reshaping the future with NFV and SDN: The Impact of New Tehnologies on Carriers and Their Networks. Available at: https://www.adlittle.com/sites/default/files/viewpoints/ADL_BellLabs_2015_Reshapingthefuture.pdf

Richter, F. (2017). Netflix Surpasses Major Cable Providers in the U.S. Available at: https://www.statista.com/chart/9799/netflixvs-cable-pay-tv-subscribers/ (Accessed: 9 June 2018).

Steingröver, M., Cardozo Larrea, E. B. and Zhelev, N. (2019). The Rise of OTT Players: What is the Appropriate Regulatory Response?', in Krüssel, P. (ed.) Future Telco: Successful Positioning of Network Operators in the Digital Age. Cham: Springer International Publishing, pp. 241-249. https://doi.org/10.1007/978-3-319-77724-5_21

The TM Forum Website. (2020). Available at: https://www.tmforum.org

TM Forum. (2012). Portfolio and Product Management, Quick Start pack: Fulfillment. Available at: https://www.tmforum.org/resources/standard/gb959-quick-start-pack-fulfillment-v0-4/

TM Forum. (2019a). Application Framework (TAM). Available at: https://www.tmforum.org/application-framework/

TM Forum. (2019b). Business Metrics. Available at: https://www.tmforum.org/strategic-program/metrics/.

TM Forum. (2019c). Frameworx. Available at: https://www.tmforum.org/tm-forum-frameworx-2/. 
TM Forum. (2019d). Information Framework (SID). Available at: https://www.tmforum.org/information-framework-sid/. 